\title{
Zu Fuß zur Arbeit statt "Friss die Hälfte“
}

Die Erhöhung der Alltagsaktivität, etwa durch Umstellung vom Auto auf aktivere Bewegungsformen, könnte sich auf Populationsebene positiv auf den Body-Mass-Index (BMI) auswirken.

\section{Hier steht eine Anzeige.}

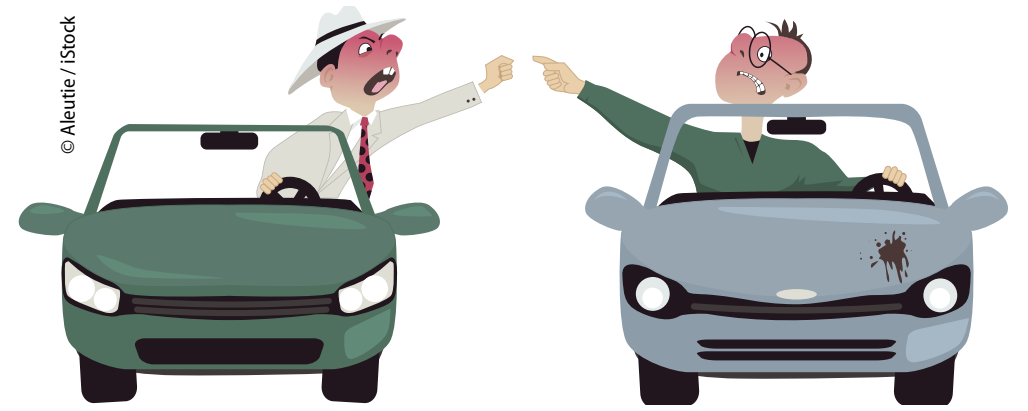

Wer mit dem Auto zur Arbeit fährt, ist viel zu oft gestresst und setzt zudem noch Speck an.

- In den Jahren 2004-2007 wurden fast 16.000 repräsentativ ausgewählte Haushalte in England zu verschiedenen Gesundheitsproblemen befragt. $\mathrm{Zu}$ den erhobenen Daten gehörten auch Größe und Gewicht zu Beginn der Untersuchung und nach zwei Jahren. Die Daten einer Untergruppe von 4.056 Haushalten wurden nun in einer multivariaten linearen Regressionsanalyse ausgewertet, um zu ergründen, wie sich der BMI in Abhängigkeit von der Art des Arbeitswegs entwickelt.

Nach Korrektur für sozioökonomische und gesundheitsbezogene Kovariablen zeigte sich, dass 3.269 Personen, die vom Auto auf öffentliche Verkehrsmittel, Fahrräder und Fußwege umgestiegen waren, ihren BMI um $0,32 \mathrm{~kg} / \mathrm{m}^{2}$ im Vergleich zu 3.090 Personen ohne diese Umstellung gesenkt hatten. Dabei ergab sich ein dosisabhängiger Effekt: Je länger der Weg zur Arbeit war, desto mehr ging der BMI zurück. 787 Personen änderten in diesem Zeitraum ihr Leben in umgekehrter Richtung: Sie wechselten von einem körperlich aktiven Weg zur Arbeit zum Auto, was sie mit einem signifikanten Anstieg des BMI um $0,34 \mathrm{~kg} / \mathrm{m}^{2}$ „bezahlten“.

- Martin A et al. Impact of changes in mode of travel to work on changes in body mass index: evidence from the British Household Panel Survey. J Epidemiol Community Health 2015;69:753-61

\section{KOMMENTAR}

Die Stärke der Studie liegt vor allem im longitudinalen Ansatz derselben Kohorte. Sie zeigt eindrucksvoll, dass für die langfristige Zu-wie auch Abnahme an Körpergewicht nicht spektakuläre kurzfristige Maßnahmen und Diätumstellungen von Bedeutung sind. Vielmehr sind es viele kleine und scheinbar unbedeutende Lebensumstände, die im Lauf von Jahren zu einer Gewichtsänderung führen. 